\title{
13 INTRODUCING ADVANCE CARE PLANNING IN COMMUNITY PALLIATIVE CARE
}

10.1136/bmjspcare-2011-000053.13

D Sives', M A Cornbleet'2, S Murray ${ }^{3}$ St Jonhs Hospital, West Lothian, Scotland; ${ }^{2}$ Strathacarron Hospice, Denny, Stirlingshire, Scotland; ${ }^{P}$ Primary Palliative Care Research Group, Edinburgh University, Edinburgh, UK

Aim To design and introduce for all patients referred to a hospice community team an intervention that facilitates advance care planning (ACP), and to evaluate the feasibility and utility of this innovation.

Design Piloting of the implementation of structured ACP, followed by a longitudinal mixed-methods evaluation. We introduced into routine practice a structured aide memoire (to facilitate ACP) and recording sheet.

We evaluated this intervention prospectively collecting detailed process and outcomes data and conducted serial interviews with patients, their case linked carers and CNS. Interviews were digitally recorded, transcribed and analysed.

Findings We routinely attempted ACP from initial contact. In only $18 \%$ of patients could this be initiated at 1 st visit due to various barriers The documentation of PPC increased compared with before (58\% vs $30 \%$ ) but the ACP prompt was only used in $8 \%$.

The serial interviews identified barriers to ACP for patients, carers and the CNS. A fear of dying and the patient perception of who should undertake ACP emerged as important patient barriers while there are specific challenges to care planning in patients with organ failure and the frail elderly with cancer.

Conclusions Facilitated ACP with prompts proved less useful for initiating conversation for CNS in palliative care. However the introduction of structured documentation improved the recording and communication of care preferences. Significant barriers to ACP exist which may persist despite skilled facilitation. More research and education is necessary before ACP can become routine for all who desire it. 